\title{
Secondary Organic Aerosol Formation from Limonene Ozonolysis: Homogeneous and Heterogeneous Influences as a Function of $\mathrm{NO}_{x}$
}

\author{
Jieyuan Zhang, Kara E. Huff Hartz, Spyros N. Pandis and Neil M. Donahue* \\ Center for Atmospheric Particle Studies, Carnegie Mellon University \\ *Corresponding Author \\ submitted to The Journal of Physical Chemistry A
}

\section{Data Tables}

Table 1: The high $\left[\mathrm{O}_{3}\right]$ experimental results in this study.

\begin{tabular}{|c|c|c|c|c|c|c|c|c|}
\hline Date & $\begin{array}{l}{[\mathrm{VOC}]_{0}} \\
(\mathrm{ppb})\end{array}$ & $\begin{array}{l}{\left[\mathrm{O}_{3}\right]_{0}} \\
(\mathrm{ppb})\end{array}$ & $\begin{array}{c}{\left[\mathrm{NO}_{x}\right]_{0}} \\
(\mathrm{ppb})\end{array}$ & $\begin{array}{c}{[\mathrm{VOC}]_{0} /\left[\mathrm{NO}_{x}\right]_{0}} \\
(\mathrm{ppbC} / \mathrm{ppb})\end{array}$ & $\begin{array}{c}\mathrm{C}_{\mathrm{OA}} \\
\left(\mu \mathrm{g} m^{-3}\right)\end{array}$ & $\mathrm{AMF}$ & 2-butanol & UV \\
\hline $11 / 10 / 05$ & 109.8 & 950 & 1400 & 0.78 & 965.7 & 1.45 & yes & on \\
\hline $11 / 14 / 05$ & 16.4 & 600 & 1400 & 0.12 & 71.4 & 0.72 & yes & on \\
\hline $11 / 16 / 05$ & 6.1 & 880 & 900 & 0.07 & 27.2 & 0.73 & yes & on \\
\hline $11 / 21 / 05$ & 5.3 & 1150 & 220 & 0.24 & 20.1 & 0.63 & yes & on \\
\hline $11 / 23 / 05$ & 7.1 & 1400 & 80 & 0.89 & 32.9 & 0.76 & yes & on \\
\hline $12 / 10 / 05$ & 3.4 & 1000 & 220 & 0.16 & 12.2 & 0.65 & yes & on \\
\hline $12 / 11 / 05$ & 1.3 & 900 & 100 & 0.13 & 3.8 & 0.51 & yes & on \\
\hline $12 / 14 / 05$ & 18.1 & 1150 & 800 & 0.23 & 146.4 & 1.33 & yes & on \\
\hline $12 / 18 / 05$ & 0.5 & 1100 & 25 & 0.22 & 0.2 & 0.06 & yes & on \\
\hline $12 / 19 / 05$ & 1.1 & 1100 & 80 & 0.13 & 1.8 & 0.29 & yes & on \\
\hline $11 / 20 / 05$ & 3.1 & 1100 & 260 & 0.12 & 4.5 & 0.23 & no & on \\
\hline $11 / 21 / 05$ & 5.6 & 1300 & 210 & 0.27 & 25.3 & 0.74 & no & on \\
\hline $12 / 16 / 05$ & 18.2 & 4500 & 100 & 1.8 & 111.0 & 1.00 & yes & on \\
\hline $2 / 1 / 06$ & 13.7 & 750 & 45 & 3.0 & 66.9 & 0.80 & yes & on \\
\hline $11 / 22 / 05$ & 6.7 & 880 & 4 & 15.0 & 18.5 & 0.45 & yes & on \\
\hline $12 / 10 / 05$ & 2.9 & 900 & 7 & 4.1 & 7.1 & 0.40 & yes & on \\
\hline $12 / 12 / 05$ & 2.0 & 1000 & 3 & 5.6 & 2.1 & 0.17 & yes & on \\
\hline $12 / 17 / 05$ & 17.5 & 3900 & 7 & 25.0 & 89.7 & 0.85 & yes & on \\
\hline $4 / 8 / 06$ & 48.5 & 800 & 2 & 243 & 242.5 & 0.82 & yes & on \\
\hline $4 / 8 / 06$ & 115.9 & 1200 & 2 & 580 & 707.6 & 1.01 & yes & on \\
\hline $11 / 10 / 05$ & 108.7 & 1500 & 5 & 217.4 & 718.4 & 1.09 & yes & off \\
\hline $11 / 14 / 05$ & 16.8 & 1100 & 5 & 33.6 & 69.9 & 0.69 & yes & off \\
\hline $11 / 19 / 05$ & 3.6 & 900 & 2 & 18.2 & 10.2 & 0.46 & yes & off \\
\hline $11 / 22 / 05$ & 6.5 & 950 & 15 & 4.3 & 25.6 & 0.65 & yes & off \\
\hline $12 / 11 / 05$ & 1.1 & 1000 & 7 & 1.5 & 1.7 & 0.26 & yes & off \\
\hline $12 / 18 / 05$ & 13.8 & 3950 & 10 & 13.8 & 79.3 & 0.95 & yes & off \\
\hline $1 / 4 / 06$ & 2.8 & 2650 & 14 & 2.0 & 8.1 & 0.48 & yes & off \\
\hline $1 / 5 / 06$ & 2.7 & 1250 & 11 & 2.4 & 6.9 & 0.42 & yes & off \\
\hline $1 / 6 / 06$ & 2.8 & 750 & 10 & 2.8 & 7.2 & 0.43 & yes & off \\
\hline $1 / 7 / 06$ & 2.8 & 220 & 7 & 4.0 & 6.4 & 0.38 & yes & off \\
\hline $2 / 2 / 06$ & 14.0 & 850 & 2 & 70.0 & 63.5 & 0.75 & yes & off \\
\hline $2 / 3 / 06$ & 4.5 & 800 & 8 & 5.6 & 14.2 & 0.52 & yes & off \\
\hline
\end{tabular}


Table 2: The low initial $\mathrm{O}_{3}$ experimental results in this study.

\begin{tabular}{|c|c|c|c|c|c|c|c|c|c|}
\hline Date & $\begin{array}{c}{[\mathrm{VOC}]_{0}} \\
(\mathrm{ppb})\end{array}$ & $\begin{array}{l}{\left[\mathrm{O}_{3}\right]_{0}} \\
(\mathrm{ppb}) \\
\end{array}$ & $\begin{array}{c}{\left[\mathrm{NO}_{x}\right]_{0}} \\
(\mathrm{ppb})\end{array}$ & $\begin{array}{c}{[\mathrm{VOC}]_{0} /\left[N O_{x}\right]_{0}} \\
(\mathrm{ppbC} / \mathrm{ppb})\end{array}$ & $\begin{array}{c}\mathrm{C}_{\mathrm{OA}} \\
\left(\mu \mathrm{g} m^{-3}\right)\end{array}$ & $\mathrm{AMF}$ & $\begin{array}{c}\mathrm{k}\left(\text { limonene }+\mathrm{O}_{3}\right) \\
\left(\mathrm{cm}^{3} \text { molecule } \mathrm{s}^{-1}\right)\end{array}$ & 2-butanol & UV \\
\hline $1 / 7 / 06$ & 58.9 & $\begin{array}{c}129 \\
1500\end{array}$ & 850 & 0.7 & $\begin{array}{l}108.0 \\
218.7\end{array}$ & $\begin{array}{l}0.30 \\
0.61\end{array}$ & $2.7 \times 10^{-16}$ & yes & on \\
\hline $2 / 4 / 06$ & 34.8 & $\begin{array}{c}85 \\
2010\end{array}$ & 240 & 1.5 & $\begin{array}{c}70.5 \\
127.9\end{array}$ & $\begin{array}{l}0.33 \\
0.61\end{array}$ & $2.9 \times 10^{-16}$ & no & on \\
\hline $1 / 23 / 06$ & 60.1 & $\begin{array}{l}115 \\
2650\end{array}$ & 20 & 30 & $\begin{array}{l}339.9 \\
349.9\end{array}$ & $\begin{array}{l}0.93 \\
0.96\end{array}$ & $1.7 \times 10^{-16}$ & yes & on \\
\hline $1 / 27 / 06$ & 56.3 & $\begin{array}{c}115 \\
2280\end{array}$ & 55 & 10.2 & $\begin{array}{l}288.6 \\
296.6\end{array}$ & $\begin{array}{l}0.84 \\
0.87\end{array}$ & $2.2 \times 10^{-16}$ & yes & on \\
\hline $1 / 29 / 06$ & 79.8 & $\begin{array}{c}110 \\
3200\end{array}$ & 80 & 10.0 & $\begin{array}{l}387.7 \\
397.5\end{array}$ & $\begin{array}{l}0.80 \\
0.82\end{array}$ & $2.3 \times 10^{-16}$ & yes & on \\
\hline $4 / 9 / 06$ & 144.6 & $\begin{array}{c}180 \\
2000\end{array}$ & 2 & 723 & $\begin{array}{l}654.4 \\
854.5\end{array}$ & $\begin{array}{l}0.92 \\
0.97\end{array}$ & $2.3 \times 10^{-16}$ & yes & on \\
\hline $1 / 19 / 06$ & 86.6 & $\begin{array}{c}240 \\
2000\end{array}$ & 2 & 433 & $\begin{array}{l}495.8 \\
520.8\end{array}$ & $\begin{array}{l}0.98 \\
0.99\end{array}$ & & yes & off \\
\hline $1 / 20 / 06$ & 56.5 & $\begin{array}{c}120 \\
2200\end{array}$ & 1 & 565 & $\begin{array}{l}231.6 \\
325.7\end{array}$ & $\begin{array}{l}0.94 \\
0.95\end{array}$ & $1.7 \times 10^{-16}$ & yes & off \\
\hline
\end{tabular}

The second line for each experiment shows the experimental conditions of the second ozone injection.

\section{$2 \quad$ UV Radiation Effect}
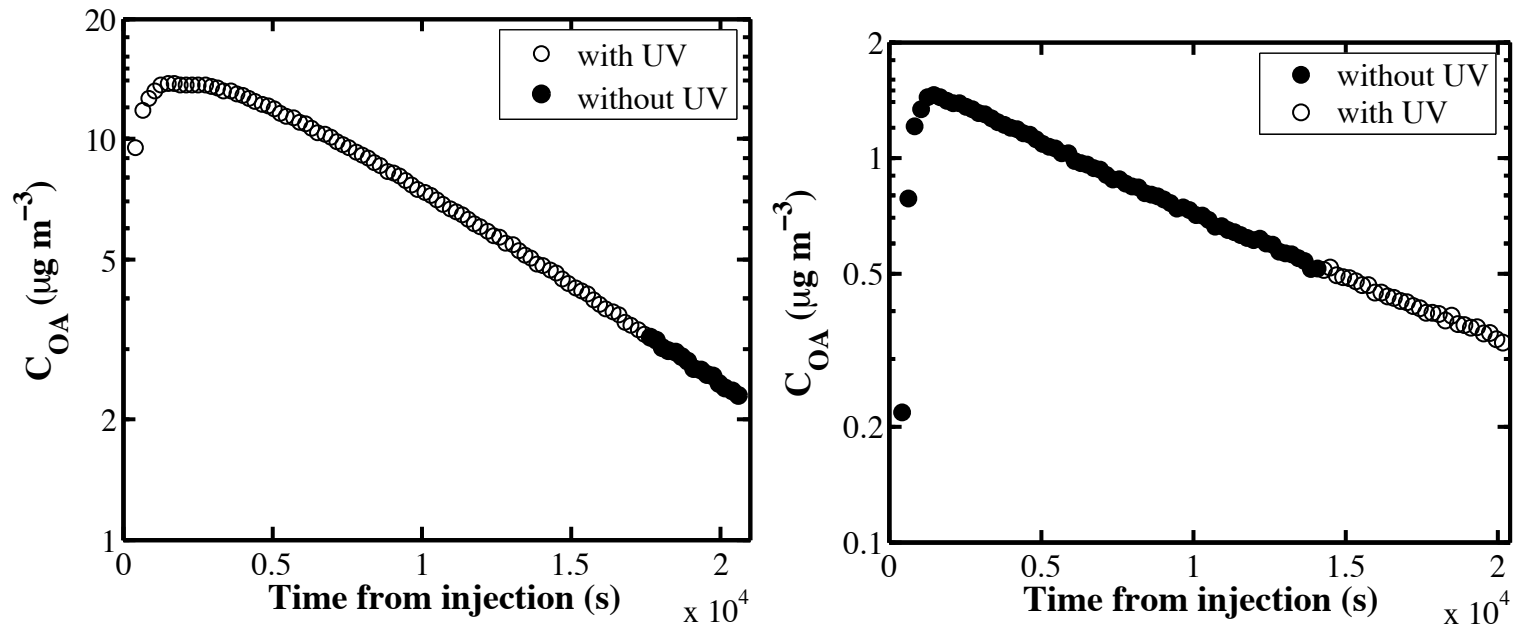

Figure 1: The SOA concentration inside the bag as a function of time for (a) UV radiation from 'on' to 'off' and (11/22/05) (b) UV radiation from 'off' to 'on' $(12 / 11 / 05)$. The Aerosol concentration is plotted in logarithmic scale, since the wall loss of aerosol is a first order process, and the aerosol concentration decreases linearly in logarithmic scale. 